\title{
Fatores preditores e qualidade de vida das vítimas de trauma por acidentes de
}

\section{trânsito}

\author{
Predicting factors and quality of life of trauma victims for traffic accidents \\ Factores predictivos y calidad de vida de las víctimas de trauma por accidentes de tráfico
}

Recebido: 01/04/2021 | Revisado: 11/04/2021 | Aceito: 13/04/2021 | Publicado: 25/04/2021

\author{
Damião da Conceição Araújo \\ ORCID: https://orcid.org/0000-0003-1116-170X \\ Universidade Federal de Sergipe, Brasil \\ E-mail: damiao.araujo92@gmail.com \\ Carla Passos Almeida \\ ORCID: https://orcid.org/0000-0002-6935-8161 \\ Universidade Federal de Sergipe, Brasil \\ E-mail: ccarlapassos@gmail.com \\ Luciana Rodrigues Prata Santana \\ ORCID: https://orcid.org/0000-0002-5074-1208 \\ Universidade Federal de Sergipe, Brasil \\ E-mail: luuh.rps@gmail.com \\ Allan Dantas dos Santos \\ ORCID: https://orcid.org/0000-0002-6529-1887 \\ Name of institution where it operates, Country \\ E-mail: allanufs@hotmail.com \\ Shirley Verônica Melo Almeida Lima \\ ORCID: https://orcid.org/0000-0002-9062-0742 \\ Universidade Federal de Sergipe, Brasil \\ E-mail: shirleymelo.lima@gmail.com \\ Karina Conceição Gomes Machado de Araújo \\ ORCID: https://orcid.org/0000-0003-4433-5227 \\ Universidade Federal de Sergipe, Brasil \\ E-mail: karinaconceicaoaraujo@gmail.com \\ José Antônio Barreto Alves \\ ORCID: https://orcid.org/0000-0002-8647-8050 \\ Universidade Federal de Sergipe, Brasil \\ E-mail: antoniobalves@gmail.com \\ Valter Joviniano de Santana Filho \\ ORCID: https://orcid.org/0000-0003-1488-0259 \\ Universidade Federal de Sergipe, Brasil \\ E-mail: vjsf@infonet.com.br \\ Andreia Centenaro Vaez \\ ORCID: https://orcid.org/0000-0002-1503-0785 \\ Universidade Federal de Sergipe, Brasil \\ E-mail: andreiacentenarovaez@gmail.com
}

\begin{abstract}
Resumo
Os acidentes de trânsito representam um importante problema de saúde pública mundial, em virtude do impacto morbimortalidade e qualidade de vida das vítimas, o presente artigo teve como objetivo avaliar os fatores preditores e a qualidade de vida das vítimas de trauma por acidentes de trânsito. Trata-se de um estudo observacional do tipo transversal com abordagem descritiva. A amostra foi não probabilística por conveniência, composta por 73 vítimas de trauma por acidentes de trânsito. A coleta de dados ocorreu em dois momentos: (1) durante a internação hospitalar; e (2) após 90 dias decorridos do evento traumático. Os instrumentos utilizados para o levantamento dos dados foi o de caracterização do perfil sociodemográfico e do acidente de trânsito, e o World Health Organization Quality of Life (WHOQOL) Bref. Os dados foram armazenados em planilhas eletrônicas do microsof excel 2013 e analisados com o programa BioEstat 5.0. Os fatores preditores que revelaram ter influência nos acidentes de trânsito, foram o sexo masculino (OR: 39.6), uso do álcool (OR: 31.7), ausência dos equipamentos de proteção individual (OR: 3.00) e uso de veículo automotor nos finais de semana (OR: 3,68$)$. Na avaliação da qualidade de vida, os achados evidenciam que no trauma o domínio físico $(35.1 \pm 14.8)$ foi o mais alterado quando comparado com os valores antes $(80.5 \pm 11.2) \mathrm{e}$

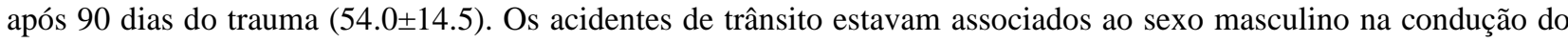
veículo, com o consumo de álcool e o não uso dos equipamentos de proteção individual. Além disso, o trauma estar relacionado ao impacto negativo na qualidade de vida das vítimas.
\end{abstract}

Palavras-chave: Causas externas; Acidentes de trânsito; Qualidade de vida. 


\begin{abstract}
Traffic accidents represent an important public health problem worldwide, due to the morbidity and mortality impact and quality of life of the victims, this article aimed to evaluate the predictive factors and the quality of life of victims of trauma due to traffic accidents. from an observational cross-sectional study. The sample was non-probabilistic for convenience, composed of 73 victims of trauma due to traffic accidents. Data collection occurred in two moments: (1) during hospitalization; and (2) after 90 days after the traumatic event. The instruments used to collect the data were the characterization of the sociodemographic profile and the traffic accident, and the World Health Organization Quality of Life (WHOQOL) Bref. The data were stored in spreadsheets of microsof excel 2013 and analyzed using the BioEstat 5.0 program. The predictive factors that revealed to have influence in the traffic accidents, were the masculine sex (OR: 39.6), alcohol use (OR: 31.7), absence of the individual protection equipment (OR: 3.00) and use motor vehicle on weekends (OR: 3.68). In assessing quality of life, the findings show that in trauma, the physical domain $(35.1 \pm 14.8)$ was the most altered when compared to the values before $(80.5 \pm 11.2)$ and after 90 days of trauma $(54.0 \pm 14.5)$. Traffic accidents were associated with men driving the vehicle, with alcohol consumption and not using personal protective equipment. In addition, the trauma is related to the negative impact on the victims' quality of life.
\end{abstract}

Keywords: External causes; Traffic-accidents; Quality of life.

\title{
Resumen
}

Los accidentes de tránsito representan un importante problema de salud pública a nivel mundial, debido al impacto de morbilidad y mortalidad y la calidad de vida de las víctimas, este artículo tuvo como objetivo evaluar los factores predictivos y la calidad de vida de las víctimas de trauma por accidentes de tránsito. estudio transversal. La muestra fue no probabilística por conveniencia, compuesta por 73 víctimas de traumatismos por accidentes de tránsito. La recolección de datos ocurrió en dos momentos: (1) durante la hospitalización; y (2) después de 90 días después del evento traumático. Los instrumentos utilizados para la recogida de datos fueron la caracterización del perfil sociodemográfico y del accidente de tráfico, y la Organización Mundial de la Salud de Calidad de Vida (WHOQOL) Bref. Los datos se almacenaron en hojas de cálculo de microsof Excel 2013 y se analizaron mediante el programa BioEstat 5.0. Los factores predictivos que revelaron tener influencia en los accidentes de tránsito fueron el sexo masculino (OR: 39.6), el consumo de alcohol (OR: 31.7), la ausencia del equipo de protección individual (OR: 3.00) y el uso de vehículo motorizado los fines de semana (OR: : 3.68). Al evaluar la calidad de vida, los hallazgos muestran que en el trauma, el dominio físico $(35.1 \pm 14.8)$ fue el más alterado en comparación con los valores antes $(80.5 \pm$ 11.2) y después de 90 días del trauma $(54.0 \pm 14.5)$. Los accidentes de tráfico se asociaron con hombres que conducían el vehículo, con consumo de alcohol y sin equipo de protección personal. Además, el trauma está relacionado con el impacto negativo en la calidad de vida de las víctimas.

Palabras clave: Causas externas; Accidentes de tráfico; Calidad de vida.

\section{Introdução}

Os acidentes de trânsito representam um problema grave e complexo de saúde pública global (Rios et al., 2019). As lesões causadas pelos acidentes de trânsito representam a nona causa de morte no mundo (Dalal et al., 2013) e a oitava causa de ano de vida ajustado por deficiência (DALY) (World Health Organisation, 2014). A média de mortes que ocorrem nas estradas do mundo chega a 1,24 milhão anualmente (Peden, 2005). Globalmente, a maioria das mortes por acidentes de trânsito ocorre em ocupantes de automóveis (31\%), veículos motorizados de duas a três rodas (23\%) e pedestres (22\%) (World Health Organisation, 2013). Os acidentes impactam no setor econômico, sobretudo nos países em desenvolvimento, em virtude do atendimento as vítimas na reabilitação e afastamento das atividades laborais (Nantulya et al., 2003).

O aumento dos acidentes de trânsito tem sido relacionado com o desenvolvimento econômico e tecnológico, bem como provocam implicações socioeconômicas, emocionais e físicas significativas, uma vez que são prevalentes em indivíduos em idade produtiva (Moreira et al., 2018). Estudos apontam que os acidentes de trânsito serão a segunda causa de morte (Rodrigues et al., 2018), variando conforme o sexo, a faixa etária e a distribuição espacial em diferentes regiões do mundo (Ferri et al., 2018; Bonfim et al., 2018).

Os fatores como o crescimento exponencial e não planejada da população, o aumento da taxa de urbanização e o aumento da frota de veículos, associados a prática de comportamento de risco no trânsito, como o uso de substâncias psicoativas, abuso de velocidade e o não uso dos equipamentos de proteção individual, influenciam na incidência e mortalidade (Sousa et al. 2018), sobrecarregando tratamento e reabilitação das vítimas. As incapacidades temporárias ou permanentes 
provocadas pelos acidentes de trânsito podem influenciar na qualidade de vida das vítimas durante a hospitalização e após o trauma (Paiva et al., 2016).

Uma forma de verificar o impacto do acidente e as incapacidades relacionadas em médio e longo prazo é analisando a Qualidade de Vida Relacionada à Saúde (QVRS). As avaliações de QVRS são subjetivas e autorrelatadas e podem incorporar construtos multidimensionais, que incluem aspectos da saúde física, estado psicológico, nível de independência, relações sociais e características do ambiente (World Health Organisation, 1993).

Destarte, objetivou-se com este estudo avaliar os fatores preditores e a qualidade de vida das vítimas de trauma por acidentes de trânsito.

\section{Metodologia}

Estudo observacional do tipo transversal com abordagem descritiva, realizado em um hospital público situado na região centro-sul do estado de Sergipe, Nordeste do Brasil, aprovado pelo Comitê de Ética em Pesquisa com Seres Humanos da Universidade Federal de Sergipe (UFS) sob protocolo CAAE nº 33657014.7.0000.5546.

A amostra foi não probabilística por conveniência, composta por 73 vítimas de trauma por acidentes de trânsito, de ambos os sexos, com idade igual ou superior a 18 anos, com ocorrência do acidente em um dos 75 municípios do estado de Sergipe e tempo de internação hospitalar igual ou superior a 24 horas. Foram excluídas as vítimas com alteração do nível de consciência, que não possuíam um familiar ou responsável para fornecer o consentimento para a pesquisa e que tivessem evadido do hospital ou transferidas para outras instituições de saúde.

A coleta de dados ocorreu em dois momentos: (1) durante a internação hospitalar; e (2) após 90 dias decorridos do evento traumático. Os pesquisadores frequentaram a unidade hospitalar durante um período de quatro meses (janeiro a maio de 2014), ininterruptamente, nos horários estabelecidos de 08h:00min às 18h:00min, nos setores hospitalares da urgência e emergência, ortopedia e clínica cirúrgica. Os prontuários das vítimas de trauma eram localizados nos setores para avaliação e aplicação dos critérios de inclusão e exclusão. Posteriormente, os pacientes eram visitados para o esclarecimento do objetivo da pesquisa, solicitação da assinatura do TCLE e aplicação dos instrumentos de coleta de dados. Transcorridos noventa (90) dias do trauma, as vítimas foram contatadas por telefone para nova coleta de dados.

Os instrumentos utilizados para o levantamento dos dados foi o de Caracterização do perfil sociodemografico e do acidente de trânsito, e o World Health Organization Quality of Life (WHOQOL) Bref (Fleck, 2000). Ressalta-se, que o questionário do WHOQOL-Bref foi aplicado para avaliar a qualidade de vida referente ao momento antes do trauma, com o trauma (internamento hospitalar) e após 90 dias do evento traumático. As variáveis estudadas foram: sexo, idade, raça/cor da pele, estado civil, atividade de trabalho, zona de moradia, dia de semana e turno da ocorrência do acidente, tipo e locomoção da vítima, uso de equipamentos de proteção individual, uso de álcool e outras drogas, região corporal atingida no trauma, alteração subjetiva da qualidade de vida.

Os dados foram armazenados seguindo processo de digitação dupla, por pesquisadores habilitados, em planilhas eletrônicas do microsof excel para evitar erros durante entrada de dados. Utilizou-se o programa BioEstat 5.0 para análise estatística. Foi realizado análise descritiva da amostra. As variáveis categóricas foram expressas por meio de frequência absoluta e relativa, e as numéricas caracterizadas pela medida de tendência central e de dispersão. Aplicou-se o teste de Kolmogorov-Smirnov para verificar a aderência à curva normal das variáveis quantitativas. O teste do Qui-quadrado de Pearson foi aplicado para verificar a correlação entre as variáveis independentes e a ocorrência do acidente de trânsito (desfecho primário). Considerou-se significativa a correlação com valor de $\mathrm{p}<0,05$.

A regressão logística múltipla foi utilizada na análise multivariada para identificar os fatores preditores na ocorrência do acidente de trânsito. Para a seleção das variáveis foi aplicado a estratégia de recuos para a construção do modelo de 
regressão. O primeiro modelo incluiu todas as variáveis do estudo. As variáveis que não possuíam valor de $\mathrm{p}<0,05$ não foram incluídas para uso no modelo permanente. O modelo final foi elaborado com as variáveis: sexo, uso de álcool, uso de drogas, uso de equipamentos de proteção individual (EPI), dia da semana e turno de ocorrência do acidente. Verificou-se a razão de chance (OR: odds ratio) e os intervalos de confiança.

A avaliação da qualidade de vida foi realizada por meio dos cálculos para determinar os escores. Os valores foram transformados numa escala de 0 a 100, sendo considerado os valores próximos a zero (0) como insatisfação e a cem (100) satisfação. Escores mais elevados indicam maior qualidade de vida (Sintaxe, 1998). A comparação da qualidade de vida antes do trauma, com o trauma e após 90 dias, foi realizada com o teste não-paramétrico de Mann-Whitney, adotando nível de significância com $\mathrm{p}<0.05$. Para verificar a relação dos domínios físicos, psicológicos, das relações sociais e do meio ambiente na determinação da qualidade de vida geral dos pacientes, foi aplicado o teste de regressão linear múltipla. Foi verificado o valor do coeficiente e o coeficiente de determinação (R2). Considerou-se como significativo $\mathrm{p}<0.05$ e R2 próximo ou igual ao valor 1.

\section{Resultados}

O perfil sociodemográfico das vítimas revelou idade média de 32.5 anos com desvio padrão de \pm 11.9 . Os dados descritos na Tabela 1 demonstram que a maioria eram indivíduos do sexo masculino (86.3\%), que autodeclararam raça/cor da pele parda (46.6\%), solteiros (48.0\%), com atividade de trabalho caracterizada como autônoma (64.4\%) e residentes em área rural $(63.0 \%)$. 
Tabela 1. Distribuição do perfil sociodemográfico das vítimas por acidentes de trânsito.

\begin{tabular}{|c|c|c|c|}
\hline Varáveis & Categorias & $\mathbf{n}$ & $\%$ \\
\hline \multirow[t]{2}{*}{ Gênero } & Masculino & 63 & 86.3 \\
\hline & Feminino & 10 & 13.7 \\
\hline \multirow[t]{5}{*}{ Raça/cor da pele } & Branca & 22 & 30.1 \\
\hline & Preta & 14 & 19.2 \\
\hline & Parda & 34 & 46.6 \\
\hline & Indígena & 1 & 1.3 \\
\hline & Amarela & 2 & 2.7 \\
\hline \multirow[t]{5}{*}{ Estado civil } & Nunca se casou & 35 & 48.0 \\
\hline & Casado & 18 & 24.6 \\
\hline & Separado/divorciado & 8 & 11.0 \\
\hline & Viúvo & 2 & 2.7 \\
\hline & Mora junto & 10 & 13.7 \\
\hline \multirow[t]{8}{*}{ Atividade de trabalho } & Remunerado & 16 & 22.0 \\
\hline & Autônomo & 47 & 64.4 \\
\hline & Não remunerado & 1 & 1.3 \\
\hline & Estudante & 4 & 5.5 \\
\hline & Dona de casa & 1 & 1.3 \\
\hline & Aposentado & 1 & 1.3 \\
\hline & Desempregado & 2 & 2.7 \\
\hline & Outros & 1 & 1.3 \\
\hline \multirow[t]{2}{*}{ Zona } & Urbana & 46 & 63.0 \\
\hline & Rural & 27 & 31.0 \\
\hline Total & & 73 & 100 \\
\hline
\end{tabular}

Fonte: Autores.

Os acidentes de trânsito ocorreram em sua maioria no domingo (39.7\%), no turno das 12:00 às 17:58 (44.0\%), com vítimas que eram condutoras $(63.0 \%)$ de motocicleta (85.5) e que se evolveram no acidente com outros condutores de moto (42.4\%). No que se refere ao comportamento de risco, as vítimas informaram fazer o uso correto dos equipamentos de proteção individual (55.0\%) e que não estavam sob efeito do álcool (85.0\%) e outras drogas (94.2\%). As extremidades (64.48\%) foram a região corporal mais atingida (Tabela 2). 
Tabela 2. Caracterização do acidente de trânsito.

\begin{tabular}{|c|c|c|c|}
\hline Varáveis & Categorias & $\mathbf{n}$ & $\%$ \\
\hline \multirow[t]{7}{*}{ Dia da semana da ocorrência } & Domingo & 29 & 39.7 \\
\hline & Segunda-feira & 7 & 9.6 \\
\hline & Terça-feira & 8 & 11.0 \\
\hline & Quarta-feira & 5 & 7.0 \\
\hline & Quinta-feira & 5 & 7.0 \\
\hline & Sexta-feira & 12 & 16.4 \\
\hline & Sábado & 7 & 9.6 \\
\hline \multirow[t]{4}{*}{ Turno da ocorrência } & $06: 00$ as $11: 59$ & 15 & 20.5 \\
\hline & $12: 00$ as $17: 59$ & 32 & 44.0 \\
\hline & $18: 00$ as $23: 59$ & 18 & 24.6 \\
\hline & $24: 00$ as $05: 59$ & 8 & 11.0 \\
\hline \multirow[t]{3}{*}{ Tipo de vítima } & Condutor & 46 & 63.0 \\
\hline & Passageiro & 19 & 26.0 \\
\hline & Pedestre & 8 & 11.0 \\
\hline \multirow[t]{4}{*}{ Locomoção da vítima } & Motocicleta & 61 & 85.5 \\
\hline & Automóvel & 4 & 5.5 \\
\hline & Bicicleta & 2 & 2.7 \\
\hline & À pé & 6 & 8.2 \\
\hline \multirow[t]{6}{*}{ Outra parte envolvida } & Motocicleta & 31 & 42.4 \\
\hline & Automóvel & 20 & 27.4 \\
\hline & Bicicleta & 1 & 1.3 \\
\hline & Caminhão & 1 & 1.3 \\
\hline & Animal & 5 & .7 .0 \\
\hline & Objeto fixo & 15 & 20.5 \\
\hline \multirow[t]{2}{*}{ Uso de EPI } & Sim & 40 & 55.0 \\
\hline & Não & 33 & 45.2 \\
\hline \multirow[t]{2}{*}{ Uso de drogas } & Sim & 2 & 2.7 \\
\hline & Não & 91 & 94.2 \\
\hline \multirow[t]{2}{*}{ Uso de álcool } & Sim & 11 & 15.0 \\
\hline & Não & 62 & 85.0 \\
\hline \multirow[t]{6}{*}{ Região corporal atingida } & Face, abdome e extremidades & 1 & 1.3 \\
\hline & Extremidades & 47 & 64.4 \\
\hline & Face e extremidades & 5 & 7.0 \\
\hline & Tórax & 7 & 9.6 \\
\hline & Superfície externa & 5 & 7.0 \\
\hline & Extremidades e cintura pélvica & 8 & 11.0 \\
\hline Total & & 73 & 100 \\
\hline
\end{tabular}


Os fatores preditores (Tabela 3) que revelaram associação nos acidentes de trânsito, foram o sexo masculino (OR: 39.6), uso do álcool (OR: 31.7), ausência dos equipamentos de proteção individual (OR: 3.00) e uso de veículo automotor nos finais de semana (OR: 3.68).

Tabela 3. Fatores preditores aos acidentes de trânsito das vítimas de trauma por acidente de trânsito.

\begin{tabular}{|c|c|c|c|c|c|c|}
\hline \multicolumn{2}{|c|}{ Variáveis/categorias } & \multicolumn{2}{|c|}{ Acidente de Trânsito } & \multirow[b]{2}{*}{ Odds Ratio* } & \multirow[b]{2}{*}{ IC 95\%* } & \multirow[b]{2}{*}{$\mathbf{p}^{*}$} \\
\hline & & $\mathbf{n}$ & $\%$ & & & \\
\hline \multirow[t]{2}{*}{ Sexo } & Masculino & 63 & 86.3 & 39.6 & $15.4-70.9$ & 0.00 \\
\hline & Feminino & 10 & 13.7 & & & \\
\hline \multirow[t]{2}{*}{ Uso de álcool } & Sim & 62 & 85.0 & 31.7 & $12.8-78.6$ & 0.01 \\
\hline & Não & 11 & 15.0 & & & \\
\hline \multirow[t]{2}{*}{ Uso de drogas } & Sim & 2 & 2.7 & 0.00 & - & 0.02 \\
\hline & Não & 91 & 97.2 & & & \\
\hline \multirow[t]{2}{*}{ Uso de EPI } & Sim & 33 & 45.0 & 3.00 & $1.37-6.54$ & 0.02 \\
\hline & Não & 40 & $\overline{55.0}$ & & & \\
\hline \multirow[t]{2}{*}{ Dia da semana } & Finais de semana & 48 & 65.7 & 3.68 & $1.69-7.30$ & 0.00 \\
\hline & Dias facultativos & 25 & $\overline{34.3}$ & & & \\
\hline \multirow[t]{2}{*}{ Turno da ocorrência } & Noturno/Madrugada & 28 & 38.0 & 0.34 & $0.7-0.66$ & 0.00 \\
\hline & Matutino/Vespertino & 47 & $\overline{62.0}$ & & & \\
\hline
\end{tabular}

*Teste de Regressão logística múltipla (p <0.05), Intervalo de confiança (IC) de 95\%. Fonte: Autores.

As médias e desvio padrão dos escores dos domínios do WHOQOL-Bref estão descritos na Tabela 4. Os achados evidenciam que no trauma, as vítimas alteraram significativamente os padrões físicos, psicológicos, das relações sociais e do meio ambiente. Importante destacar que o domínio físico $(35.1 \pm 14.8)$ foi o mais alterado quando comparado com os valores antes $(80.5 \pm 11.2)$ e após 90 dias do trauma $(54.0 \pm 14.5)$. 
Tabela 4. Distribuição da média e desvio-padrão dos escores por domínio do WHOQOL-Bref.

\begin{tabular}{llllll}
\hline Variável/categoria & \multicolumn{4}{l}{ Domínios do WHOQOL-BREF } & \\
\cline { 2 - 5 } & \multicolumn{1}{l}{ Físico } & Psicológico & Relações Sociais & Meio Ambiente \\
\cline { 2 - 5 } & Média \pm dp & Média \pm dp & Média \pm dp & Média \pm dp & \\
& & & & p $\dagger$ \\
\hline QV antes do trauma & $80.5 \pm 11.2$ & $73.8 \pm 11.7$ & $74.3 \pm 10.8$ & $63.3 \pm 8.3$ & $<0.01$ \\
QV no trauma & $35.1 \pm 14.8$ & $57.6 \pm 12.7$ & $57.9 \pm 17.5$ & $52.1 \pm 10.8$ & $<0.01$ \\
QV após 90 dias & $54.0 \pm 14.5$ & $66.8 \pm 15.2$ & $70.3 \pm 12.8$ & $59.9 \pm 12.3$ & $<0.01$ \\
\hline
\end{tabular}

$\dagger$ Teste de Qui-quadrado de Pearson ( $\mathrm{p}<0.05)$. Fonte: Autores.

A comparação da qualidade de vida das vítimas de trauma nos três momentos de avaliação demonstra uma diminuição no evento traumático e internamento hospitalar ( $\mathrm{p}<0,02)$. Após 90 dias, em razão do processo de reabilitação, observou-se um aumento significativo, mas não equivalente a qualidade de vida antes do acidente de trânsito (Figura 1).

Figura 1. Distribuição da média, desvio padrão, máximo e mínimo da qualidade de vida geral do WHOQOL-Bref, em relação as vítimas por acidentes de trânsito, antes do evento traumático, com o trauma no internamento hospitalar, e pós-trauma no período de 90 dias com a alta hospitalar. Aplicado teste não-paramétrico de Mann-Whitney (p <0.01).

Comparação da qualidade de vida das vítimas de trauma

Escore

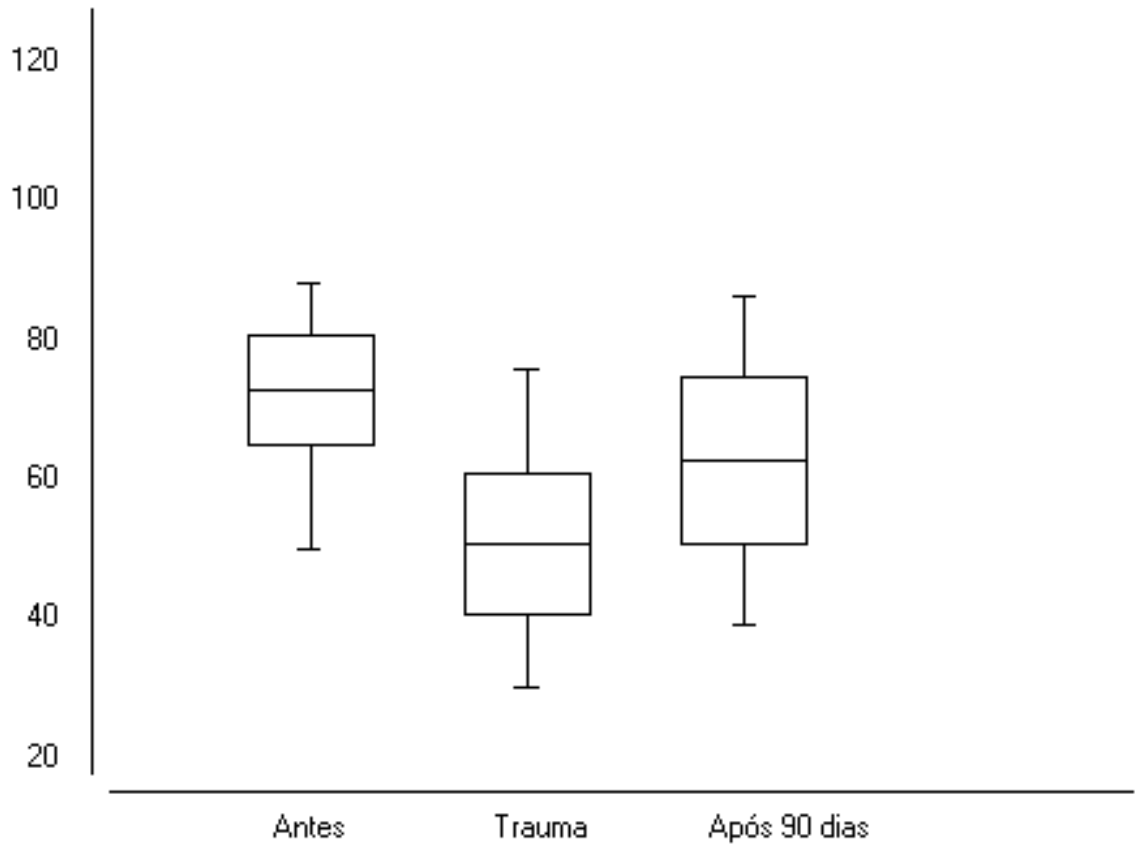

Fonte: Autores.

Na Tabela 5, a regressão linear múltipla mostra que os domínios do WHOQOL-Bref, em ambas as avaliações, possuem associação com qualidade de vida geral considerada pelas vítimas ( $\mathrm{p}<0.01)$. Contudo, é importante considerar que os valores do coeficiente dos domínios da qualidade de vida no trauma e após 90 dias representam fatores preditores com a alteração na qualidade de vida geral, uma vez que os escores estiveram abaixo do considerado ideal. 
Tabela 5. Regressão linear múltipla dos domínios do WHOQOL-Bref em relação a qualidade de vida geral da vítima de trauma nos três momentos de avaliação.

\begin{tabular}{|c|c|c|c|}
\hline Varável/categoria & Coeficiente* $^{*}$ & $\mathbf{R}^{2}$ & $\mathbf{p}^{*}$ \\
\hline \multicolumn{4}{|c|}{ Qualidade de vida antes do trauma } \\
\hline Domínio físico & 0.2496 & 0.65 & $<0.01$ \\
\hline Domínio psicológico & 0.2509 & & \\
\hline Domínio das relações sociais & 0.2502 & & \\
\hline Domínio do meio ambiente & 0.2500 & & \\
\hline \multicolumn{4}{|l|}{ Qualidade de vida no trauma } \\
\hline Domínio físico & 0.2503 & 1.0 & $<0.01$ \\
\hline Domínio psicológico & 0.2498 & & \\
\hline Domínio das relações sociais & 0.2500 & & \\
\hline Domínio do meio ambiente & 0.2497 & & \\
\hline \multicolumn{4}{|l|}{ Qualidade de vida após 90 dias } \\
\hline Domínio físico & 0.2494 & 0.75 & $<0.01$ \\
\hline Domínio psicológico & 0.2502 & & \\
\hline Domínio das relações sociais & 0.2501 & & \\
\hline Domínio do meio ambiente & 0.2501 & & \\
\hline
\end{tabular}

*Teste de Regressão linear múltipla $(\mathrm{p}<0,05)$. $\mathrm{R}^{2}$ : coeficiente de determinação. Fonte: Autores.

\section{Discussão}

Os resultados deste estudo demonstraram que as vítimas de trauma por acidentes de trânsito, em sua maioria, eram adultos jovens, do sexo masculino, que tiveram como principal tipo de acidente a queda de moto com ocorrência no final de semana. A região corporal mais atingida foram as extremidades. Os fatores preditores associados aos acidentes de trânsito foram o sexo masculino, uso do álcool, ausência dos equipamentos de proteção individual e uso de veículo automotor nos finais de semana.

A prevalência de indivíduos do sexo masculino e adultos jovens envolvidos em acidentes é relatado em outros estudos (Soliman et al., 2018; Cordellieri et al., 2016) e pode estar associado a prática de comportamento de risco na condução do veículo automotor com agressividade no trânsito, superestimação da capacidade e dos limites, experiência e habilidade de dirigir reduzida, consumo do álcool e outras drogas, o ausência de uso do capacete ( Lemos et al., 2019; Araújo et al., 2017; Barreto et al., 2016; Souto et al., 2012; Oliveira et al., 2020) e a condução do veículo sem antes ter passado por treinamento para retirar a carteira de habilitação ( Araújo et al., 2016) contribuem para o aumento do risco de acidentes e impactam na incidência e mortalidade.

O consumo de álcool na condução do veículo é fator de risco importante, uma vez que altera a percepção, visão, reflexos, consciência e comportamento dos indivíduos (Cunha et al., 2011). No Brasil, uma pesquisa demonstrou que a condução de veículo por motoristas após consumo de bebida alcóolica apresentou alta prevalência, variando de $15,7 \%$ no ano de 2011 para 11,4\% no ano de 2018. Verificou-se também maior ocorrência entre homens e adultos mais jovens ${ }^{25}$.

O uso do capacete é obrigatório para condutores e passageiros de moto. Contudo, ainda se observa que motociclistas não aderem o uso (Besse et al., 2018). Fator preocupante, uma vez que o uso do capacete pode diminuir o risco de lesões cranioencefálicas e morte. Desta forma, é recomendado a fiscalização para o cumprimento da lei por serviços de vigilância das rodovias para prevenir esse comportamento de risco (Bao et al., 2017). 
Outros fatores que favorecem os acidentes de trânsito incluem os relacionados ao ser humano como: erro, comportamento e fadiga do motorista, comportamento do motorista, excesso de velocidade, uso de celulares durante a condução, mudança repentina de faixas, ignorância sobre regras e regulamentos de trânsito, erros de travessias das rodoviárias; e os fatores ambientais como pneus e luzes defeituosos, condições das estradas, fluxo de tráfego e congestionamento, condições climáticas, entre outros (Souza et al., 2018).

$\mathrm{O}$ envolvimento de motocicletas nos acidentes de trânsito evidencia o alto impacto desse veículo automotor em vítimas de trauma. As motocicletas enfrentam maiores perigos nas estradas do que carros ou outros veículos, em razão do tamanho ser menor e possuir instabilidade no enfrentamento de aspectos da natureza e infraestrutura das vias de trânsito como buracos, animais mortos ou que trafeguem nas vias, as condições da pavimentação, irregularidades das pistas e a presença de outros objetos inesperados na estrada, representando uma ameaça grave para a segurança dos condutores de moto (Sousa et al., 2021).

A qualidade de vida é definida pela Organização Mundial de Saúde (OMS) como "a percepção da pessoa sobre sua posição na vida dentro do contexto dos sistemas culturais e de valores nos quais ela vive e em relação a suas metas, expectativas pessoais e preocupações" (World Health Organization, 2018).

Os escores do WHOQOL-bref, neste estudo, revelaram que antes do trauma as vítimas consideraram sua qualidade de vida satisfatória. No entanto, com o trauma e o internamento hospitalar, houve alteração significativa em relação aos aspectos da qualidade de vida. Destaca-se que mesmo após transcorridos 90 dias do acidente, as vítimas ainda possuíam escores baixo para a qualidade de vida global e os respectivos domínios. A associação de cada domínio do WHOQOL-bref como fator de risco para qualidade de vida global, revelou que no trauma o domínio físico foi o que mais impactou, uma vez que o trauma desencadeia diversas alterações fisiológicas em virtudes das lesões como dor, diminuição da mobilidade física, limitação das atividades diárias e instrumentais (Bilgin et al., 2020).

Os domínios psicológico e das relações sociais também apresentaram forte correlação no momento do trauma. As vítimas por necessitarem da hospitalização e reabilitação são afastadas do convívio social e atividades laborais, o que impacta significativamente a condição psicológica. Transcorridos 90 dias do trauma, foi observado que a qualidade de vida global e os domínios acresceram os valores, demonstrando melhora na percepção da qualidade de vida. Após esse período algumas vítimas recebem alta hospitalar, bem como apresentam melhora na sua saúde física e emocional (Filho, 2016).

\section{Conclusão}

Os acidentes de trânsito foram associados ao sexo masculino na condução do veículo, com o consumo de álcool e o não uso dos equipamentos de proteção individual. Além disso, o trauma estar relacionado ao impacto negativo na qualidade de vida das vítimas no momento do trauma e depois de 90 dias do acidente. Os resultados deste estudo fornecem subsídio para direcionamento de políticas públicas e estratégias de prevenção, bem como para a realização de outros estudos na temática.

\section{Referências}

Araújo, D. C. et al. (2017). Perfil e fatores associados ao trauma em vítimas de acidentes de trânsito atendidas por serviço móvel de urgência. Arquivos de Ciências da Saúde, 24(2), 65-70.

Araújo, D. C., Vaez, A. C., Alves, J. A. B., Pinheiro, F. M. S. \& Fakhouri, R. (2016) Suicidio inconsciente: reflexo do comportamento de risco no trânsito. Rev enferm UFPE on line. 10(11), 3823-30.

Barreto, M. S., Tenson, E. F., Lattore, M. R. D. O., Mathias, T. A. F. \& Marcon, S. S. (2016). Mortalidade por acidentes de trânsito e homicídios em Curitiba, Paraná, 1996-2011. Epidemiol Serv Saude. 25(1), 95-104.

Bao, J., Bachani, A. M., Viet, C. P., Quang, L. N., Nguyen, N. \& Hyder, A. A. (2017). Trends in motorcycle helmet use in Vietnam: results from a four-year study. Puplic Health[Internet]. 144, S39-44. 
Besse, M. et al. (2018). Accidentes de moto: costo médico/económico en un hospital municipal de la ciudad de Buenos Aires. Medicina (B. Aires)[Internet]. 78(3):158-62

Bilgin, N. G., Mert, E. \& Sezgin, M. (2012). Evaluation of the effects of disabilities due to traffic accidents on the quality of life using SF-36 health survey. Acta Orthopaedica et Traumatologica Turcica; 46(3).

Bonfim, C. V., Silva, A. G. S., Araújo, W. M., Alencar, C. \& Furtado, B. M. A. (2018). Analysis of the spatial distribution of road accidents attended by the Mobile Emergency Service (SAMU-192) in a municipality of northeastern Brazil. Salud Colect. 14(1):65-75

Cordellieri, P., Baralla, F., Ferlazzo, F., Sgalla, R., Piccardi, L. \& Giannini, A. M. (2016). Gender effects in young road users on road safety attitudes, behaviors and risk perception. Frontiers in psychology. 7:1412.

Cunha, F. V., Julien, T. M. S. \& Santos, J. C. S. (2011). Caracterização dos acidentes de trânsito na cidade de Guaratinguetá, Brasil, envolvendo motocicletas. J Nurs UFPE online. 5(5): 1112-20.

Dalal, K., Lin, Z., Gifford, M. \& Svanström L. (2013). Economics of Global Burden of Road Traffic Injuries and Their Relationship with Health System Variables. Int. J. Prev. Med. 4:1442-50.

Ferri, E. et al. (2018). Atendimento de Urgência ao Paciente Vítima de Trauma - Diretrizes Clínicas. Governo do Estado do Espírito Santo - Secretaria de Estado da Saúde.

Filho, M. M. (2016). Quality of life and self-esteem in traffic victims in physical therapy. Fisioter. mov. 29(4 ): 703-712.

Fleck, M. P. A. (2000). O instrumento de avaliação de qualidade de vida da Organização Mundial da Saúde (WHOQOL-100): características e perspectivas. Ciênc saúde coletiva. 5(1):33-8.

Lemos, F. H. G., Pinto, I. M. B. S. \& Rita, L. P. S. (2019). Public policies for traffic accidents reduction: Multivariate Analysis At Br-101 Highway In Alagoas. Revista de Políticas Públicas, 23(1).

Malta, D. C., Bernal, R. T. I et al. (2020). Tendência temporal da prevalência de indicadores relacionados à condução de veículos motorizados após o consumo de bebida alcoólica, entre os anos de 2007 e 2018. Rev. bras. epidemiol. 23

Moreira, M. R.; Ribeiro, J. M.; Motta, C. T. \& Motta, J. I. J. (2018). Mortality by road traffic accidents in adolescents and young people, Brazil, 1996-2015: will we achieve SDG 3.6? Ciência \& Saúde Coletiva, 23(9):2785-27968.

Nantulya, V. M. \& Reich, M. R. (2003). Equity dimensions of road traffic injuries in lowand middle-income countries. Inj. Control Saf. Promot. 10:13-20.

Oliveira, J. B., Kerr-Corrêa, F., L, I. C., Vitti Junior, W., Nunes, H. R. C. \& Lima, M. C. P. (2020). Alcohol use and risk of vehicle accidents: cross-sectional study in the city of São Paulo, Brazil. Sao Paulo Med. J. [Internet]. 138(3): 208-215.

Organisation, W. H. (2013). Global status report on road safety: supporting a decade of action. https://www.who.int/ violence_injury_prevention/road_safety_status/2013/en/.

Organisation, W. H. (2014). Global health estimates 2014 DALY by cause, age and sex $2000-2012$. https://www.who.int/healthinfo/global_burden_disease/GHE_DALY_Global_2000_2012.xls.

Paiva, L. et al. (2016). Health status and the return to work after traffic accidents. Rev Bras Enferm. 69(3):416-22.

Peden, M. (2005). Global collaboration on road traffic injury prevention. Int. J. Inj. Contr. Saf. Promot. 12:85-91.

Rios, P. A. A., Mota, E. L. A., Ferreira, L. N., Cardoso, J. P., Santos, G. J. \& Rodrigues, T. B. (2019). Acidentes de trânsito com condutores de veículos: incidência e diferenciais entre motociclistas e motoristas em estudo de base populacional. Rev. bras. epidemiol. 22.

Rodrigues, T. S. et al. (2018). Caracterização das vítimas de acidentes de trânsito por motocicletas atendidos em um hospital de referência. Rev Interdisciplinar. 11(1)

SINTAXE SPSS. WHOQOL-bref Questionnaire. (1998). https://goo.gl/83LYp66.

Soliman, A., Alhajyaseen, W., Alfar, R. \& Alkaabi, I. (2018). Changes in Driving Behavior Across Age Cohorts in an Arab Culture: the Case of State of Qatar. Procedia Computer Science. 130:652-9.

Sousa, C. L. et al. (2018). Motorcyclical accidents: profile of victims associated by mobile urgency service. Temas em Saúde.

Souto, C. C. et al. (2016). Perfil das vítimas de acidentes de transporte terrestre relacionados ao trabalho em unidades de saúde sentinelas de Pernambuco, 2012 - 2014. Epidemiol. Serv. Saúde. 25(2), 351-361.

Souza, S. T. \& Oliveira, M. P. S. L. (2018). Análise de segurança viária que alvitrar dispositivos de segurança para amenização de acidentes de trânsitos. Revista Científica Semana Acadêmica.

Sousa, D. L., Silva, K. N. G., Ferreira, E. \& Morais, F. R. S. (2021). Incidência de lesões em motociclistas praticantes de trilhas. Rev. bras. ortop. 55(6).

World Health Organisation. (1993). Study protocol for the World Health Organization project to develop a Quality of Life assessment instrument (WHOQOL). Qual Life Res. 2(2):153-9.

World Health Organization. (2018). Global status report on road safety 2018. WHO TEAM Social Determinants of HealtH. 